\title{
Endoscopic Resection of Large Colorectal Lesions
}

\author{
David Martínez Ares, \\ Pamela Estévez Boullosa and José Ignacio Rodríguez Prada \\ Gastroenterology Department, \\ Complejo Hospitalario Universitario de Vigo, \\ Spain
}

\section{Introduction}

The colorectal cancer (CRC) is one of the most common tumors in Western countries. It is the most common gastrointestinal tumor and it is estimated that between 5 and $6 \%$ of the population will suffer it along their lives. Colorectal cancer is a tumor in which it is possible to perform primary and secondary prevention. Primary prevention consists of all actions aimed to prevent the development of cancer or precancerous lesions, whereas secondary prevention is the detection and removal of these premalignant lesions, avoiding then the development of colorectal cancer (CRC).

CRC is a disease with a well-known natural history, fulfilling in most cases the adenomacarcinoma sequence (Figure 1), and its natural history is long enough so it can be interrupted by various diagnostic and therapeutic strategies. In fact, in sporadic CRC, which represents most cases, the time since the appearance of a polyp in a colon without neoplastic disease, the posterior growing and degeneration of the lesion to finally become an invasive cancer, is usually not lower than 10-15 years. We therefore have a long time, enough to detect and remove these lesions, interrupting then the natural history of the disease and preventing the development of CRC; so endoscopic polypectomy is a therapeutic key point in the prevention of colorectal carcinoma.

Not all polyps degenerate into cancer and, consequently, not all colonic polyps should be removed. Although we already have endoscopic technology that allows us to accurately differentiate neoplastic from hyperplastic polyps, the standard practice is to remove all polyps. Definitely the result of histological examination of the polyp is which determines the way to approach in the future. The technique for the removal of polyps depends on several factors, such as size, location and morphology of the lesions. We can solve most pedunculated and sessile polyps with a simple polypectomy. Most colonic polyps are small and pedunculated, and its removal becomes relatively easy. When sessile polyps are located in the right colon due to the reduced thickness of the colonic wall in this location, injection of drugs into the submucosa before polypectomy decreases the risk of perforation. In large pedunculated polyps with thick pedicles, it can be helpful to use techniques to prevent bleeding after colonic polypectomy. Submucosal adrenaline injection at the base of the polyp or the placement of endoloop or hemostatic clips on the pedicle can prevent the development of bleeding complications. But this is not the aim of this work. Then we would 
try to describe in a practical way the different techniques for treatment of large sessile or flat lesions that can be found in the colon.

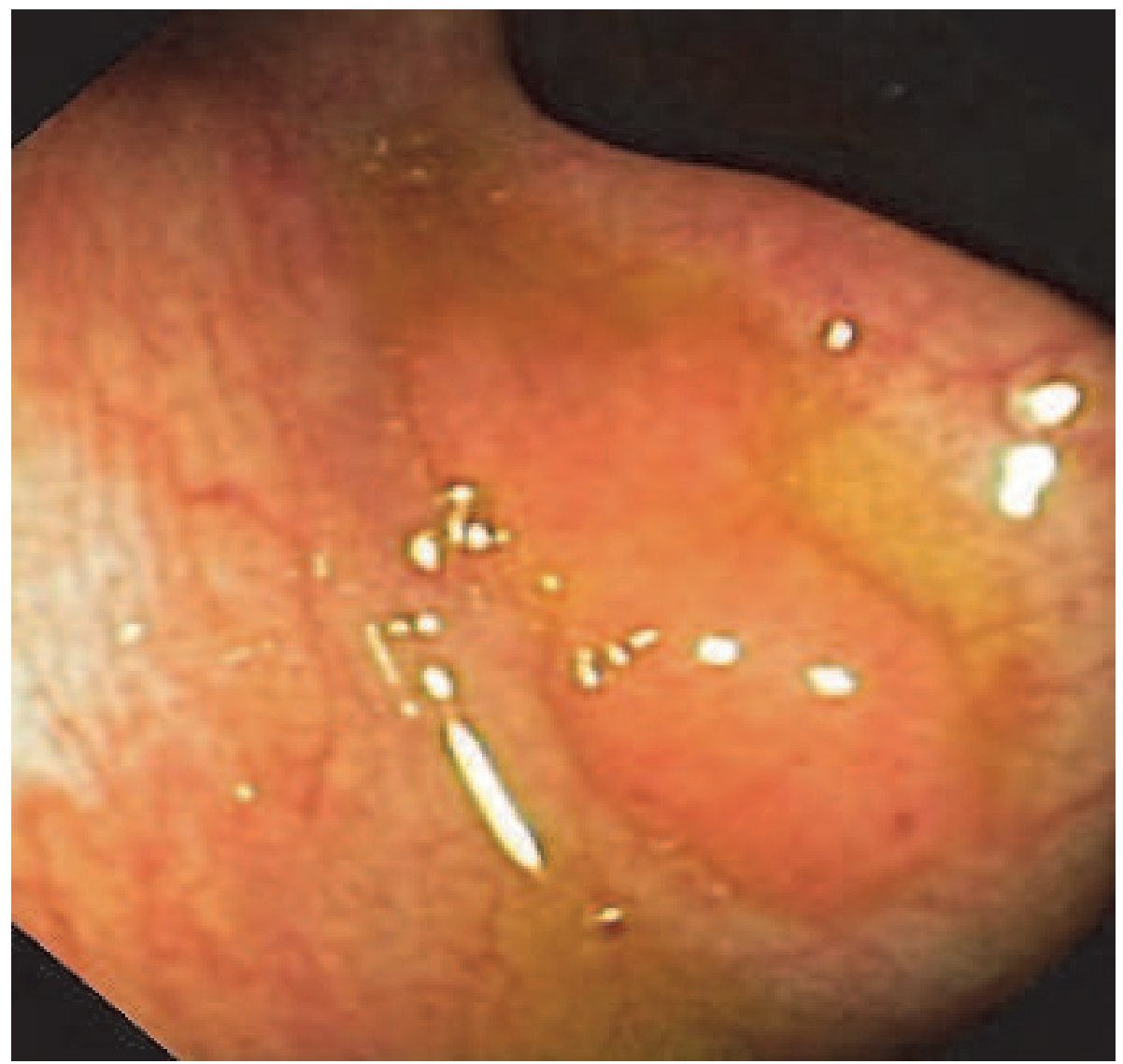

Fig. 1. The picture shows a conventional endoscopy where a flat lesion, slightly elevated and with indistinct edges can be observed.

We consider that sessile or flat lesions are large when they exceed $20 \mathrm{~mm}$ in diameter and giant polyps would be those whose size exceeds $30 \mathrm{~mm}$. As well as polyps smaller than $2 \mathrm{~cm}$ can be removed en bloc in most cases, those larger than $2 \mathrm{~cm}$ are not always achieved, and lesions larger than $3 \mathrm{~cm}$ are almost never achieved. It is accepted that lesions of this size or greater should not be removed on a single piece using a polypectomy snare. The risk of perforation is extremely high in these cases so it is recommended to perform a piecemeal resection of these lesions. The performance of this technique to achieve success, understanding it as a complete resection of the lesion, the absence of recurrence of the lesion, and the absence of complications, requires the knowledge and practice of a number of endoscopic skills that we would describe below. In many centers, these large lesions are considered in most cases for surgical treatment. We should not forget that surgery, even in case of laparoscopic surgery, involves a large increase of health costs and it is not free of 
morbidity and mortality. We should also consider that many of the patients in whom these lesions are detected have an advanced age and high comorbidity, which increases the surgical risk. On the other hand, the use of CRC screening programs makes it more frequent to diagnose this type of lesions. Therefore, adopting therapeutic strategies with lower risk to patients, lower consumption of economic resources, greater respect to organ's functionality and similar outcomes for patients seem to be more than reasonable. Naturally, we must correctly select the cases that can be solved endoscopically, avoiding then unnecessary risks and consumption of resources that will not prevent to refer patients to other interventions. Finally, treatment options that can be offered to an individual patient depend on the possibilities of each place. In very large series from reference hospitals where there are expert endoscopists show that up to two thirds of the benign lesions that are referred to surgery can be endoscopically resolved.

\section{Endoscopic resection of large colorectal lesions}

\subsection{Endoscopic Mucosal Resection (EMR)}

As it is well known this is a technique that is not exceedingly challenging from the endoscopic point of view, with little risk for complications but, rarely, it may allow removing en bloc lesions larger than $2 \mathrm{~cm}$ in diameter. However, as we will explain below, we believe that it does have an important role in colorectal neoplastic disease. It should not be, of course, a universal technique, but it would have its indications based on its benefits and its limitations.

\subsubsection{Detection and characterization of the lesion}

Nonpolypoid lesions are those consisting of dysplastic tissue that does not protrude or that protrudes a little into the intestinal lumen, reaching a maximum thickness of two times that of the surrounding mucosa. Flat lesions are often undetectable with conventional endoscopy, being essential to use endoscopic techniques such as chromoendoscopy (Figure 2), virtual chromoendoscopy or Narrow Band Imaging for the correct demarcation of the lesions. The nonpolypoid lesions of the colon can be flat and reach large dimensions but instead of growing into the intestinal lumen, they expand along the colonic wall, and they are called laterally spreading tumors (LST). These lesions have an invasive growth risk significantly lower than sessile lesions of the same size. Initially, what differentiates them it is its appearance, but there are great differences regarding their biological behavior, so in both cases, the therapeutic attitudes may be slightly different. This is, quite often, the presentation form of the lesions diagnosed in patients with long-standing ulcerative colitis and in the context of Lynch syndrome.

In order to plan the most appropriate therapeutic approach for a particular lesion we should correctly characterize it. According to most authors there are three aspects to consider in order of predicting the degree of invasion of the lesion. Some authors support performing whenever it is possible, an echoendoscopic study with high frequency miniprobes to evaluate the involvement of the colonic wall by the neoplasm. Other authors argue that endosonographic study is not essential since endoscopic appearance can greatly predict the degree of invasion of the lesion. First, the general appearance of the lesion must be defined, being strongly recommended the use of the Paris classification of superficial neoplastic lesions (2003). Most superficial endoscopic lesions are classified according to type 0 . Polypoid lesions are subtype 0I (including two variants: pedunculated (0Ip) and sessile 
(0Is)). Non-polypoid lesions are classified in subtype 0II (slightly elevated 0IIa, completely flat $0 \mathrm{IIb}$ and slightly depressed without ulcer 0IIC). Finally, subtype OIII represents nonpolypoid lesions with a frank ulcer. The risk of malignant degeneration of the lesion depends on the existence of submucosa invasion, being the maximum risk in subtype III and the minimum risk in type I lesions. Thus, those benign lesions (flat adenomas and laterally spreading tumors) and adenocarcinoma IIa lesions with a diameter less than $20 \mathrm{~mm}$ and IIb and IIc lesions less than $10 \mathrm{~mm}$. can be subsidiaries of endoscopic treatment. In lesions classified as subtype III (ulcerated) will never be indicated endoscopic treatment, so surgery would be unavoidable.

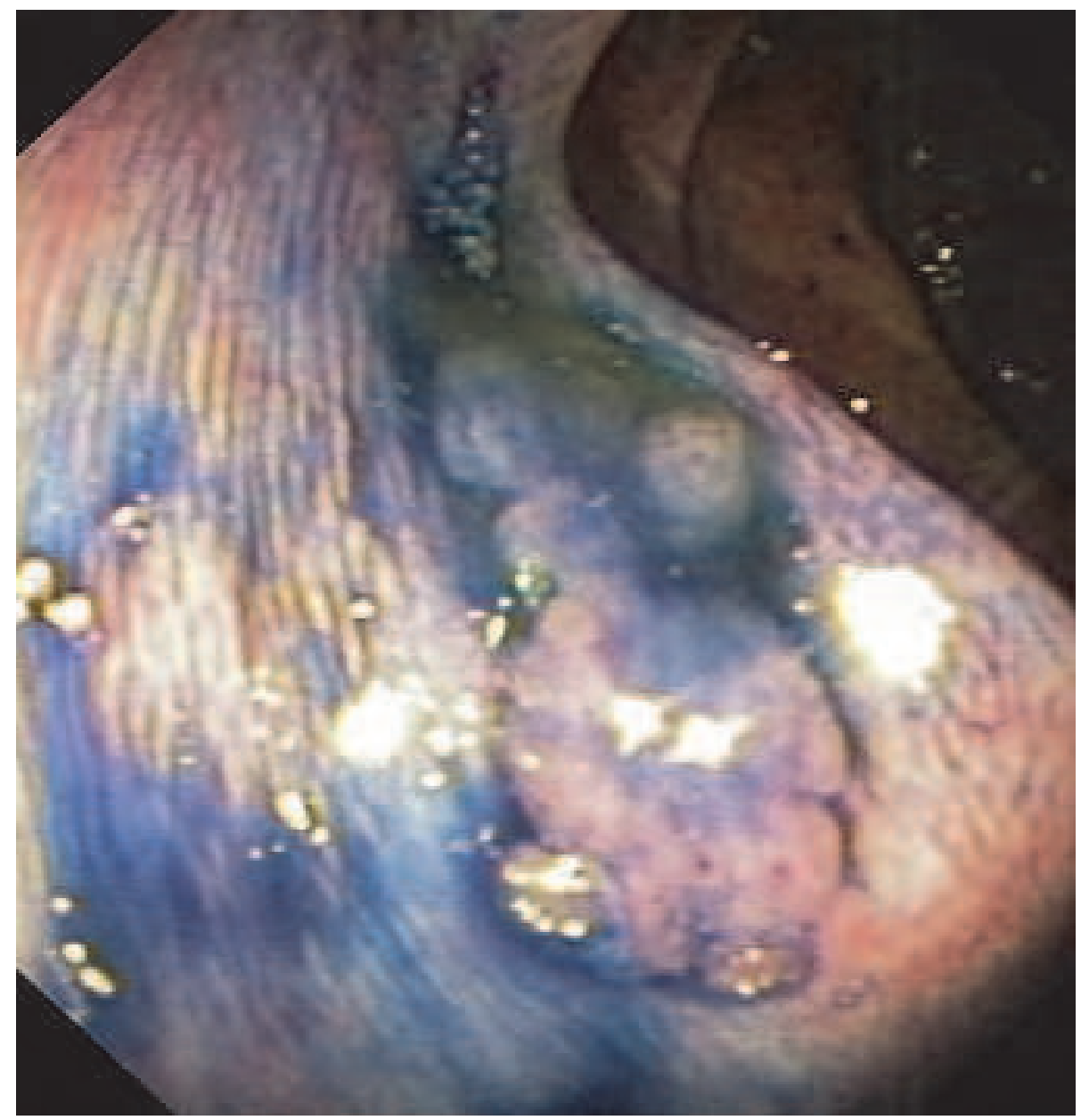

Fig. 2. Staining with indigo carmine $0.2 \%$ shows in greater detail the surface of the lesion with well defined edges.

Secondly, the appearance of the surface of the lesion has also a great transcendence, especially in LST. These LST are classified into two types according to their morphology, 
granular type (LST-G) (figure 3) and non-granular type (LST-NG) (Figure 4). LST-G lesions may reach large dimensions without presenting invasive growth, by contrast, this fact is much more likely in LST-NG. Therefore, endoscopic piecemeal mucosal resection (that is a lower complex technique and that is more available for any endoscopist) can be a therapeutic strategy for LST-G and en bloc resection with endoscopic submucosal dissection (ESD) should be applied to LST-NG, which is technically much more complex and with an increased risk of complications.

Finally, the study of crypts patterns according to Kudo's classification using chromoendoscopy and magnification endoscopy has shown a great accuracy in predicting the invasive behavior. This classification states that: type I crypt pattern consists of regular round crypts, type II consists of stellar or papillary crypts, type IIIL consists of large roundish or tubular pits, type IIIS consists of small roundish or tubular pits, type IV consists of sulcus, branch, or gyrus-like crypts, and type V consists of irregular or severely distorted crypts. Types I and II are non-neoplastic (hyperplasic lesions) and excepting some circunstances they do not require treatment. Type IIIL, IIIS and IV are non-invasive lesions so endoscopic removal can be performed and type $\mathrm{V}$ represents invasive lesions so surgical resection is the best choice for treatment.

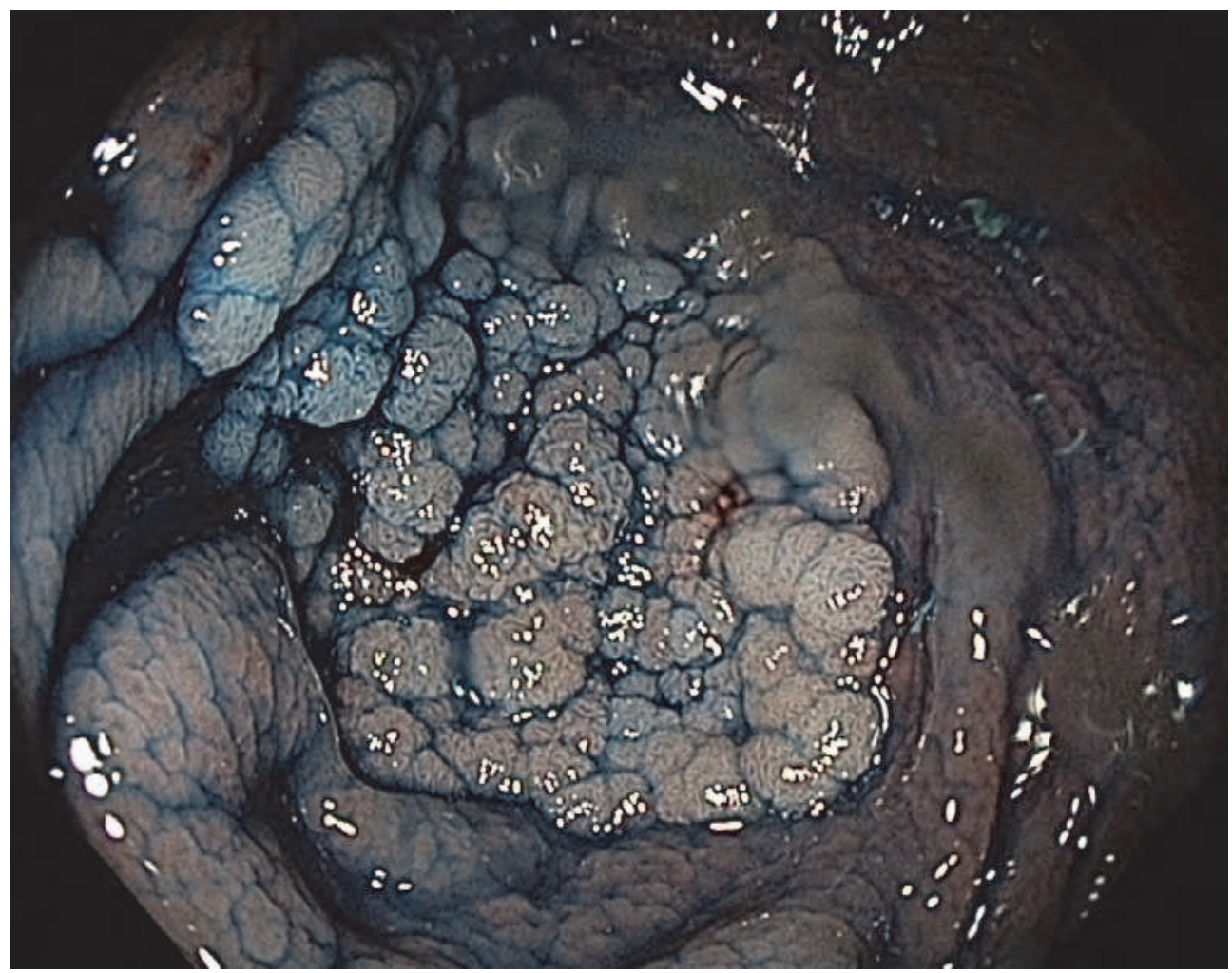

Fig. 3. Laterally spreading tumor with granular surface. It is classified as LST-G, and after its endoscopic resection a villous adenoma with low grade dysplasia was confirmed. 


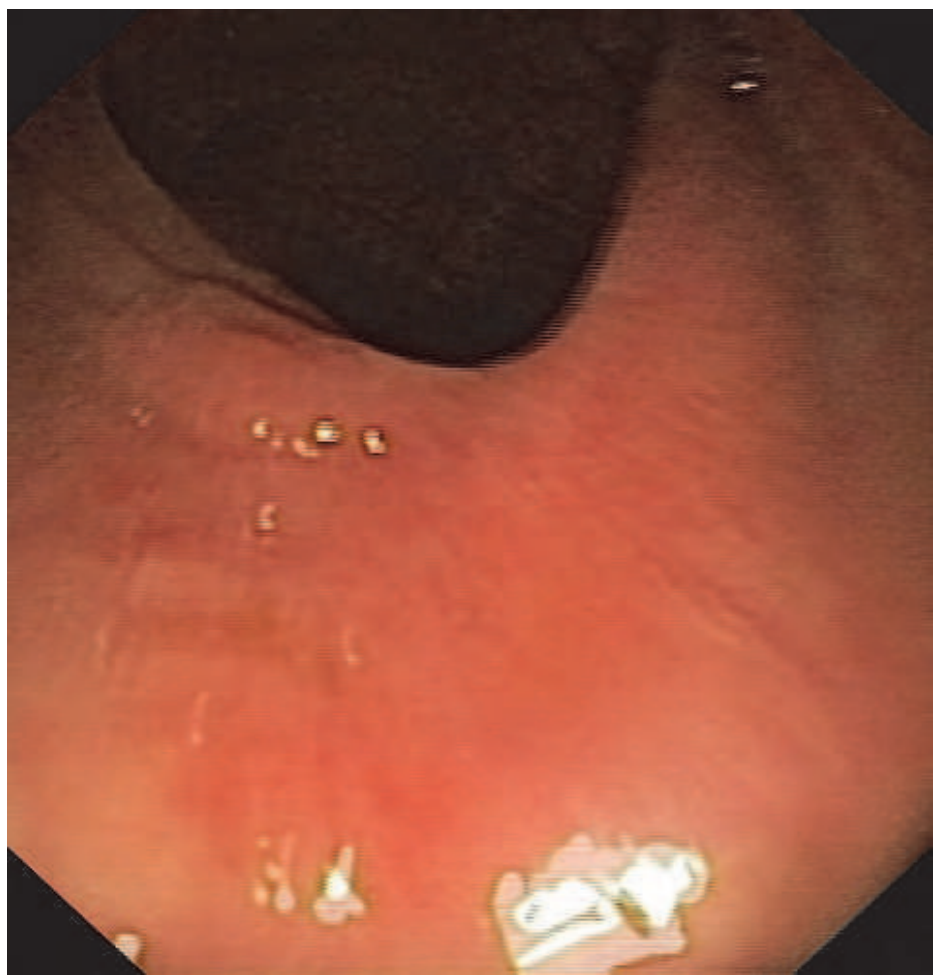

Fig. 4. Laterally spreading tumor with a smooth surface, not granular. Surgical resection was refused and the histological analysis revealed the presence of an invasive adenocarcinoma over a villous adenoma.

In conclusion, the characteristics of the lesion determine the correct treatment options: surgical or endoscopic resection. LST-NG lesions have twice the risk of presenting invasive growth, especially if pit pattern is suggestive of malignancy Kudo (V), lesions are greater than $20 \mathrm{~mm}$ of diameter and sclerotic changes are evident in the intestinal wall close to the lesion. In these cases it may be indicated surgical treatment, or in any case, a technique that allows "en bloc" resections of the lesion. In LST-G, the probability of invasive growth is increased if nodular areas larger than $10 \mathrm{~mm}$ or depressed areas are present or if the sign called "no lifting" after submucosal injection is present. So, LST-G have an infiltrative growth in less than $6 \%$ of cases, and if this takes place, more than $84 \%$ of the lesions present large nodular areas. In the absence of these data, piecemeal resection, technically easier, can be a good choice. There are some other endoscopic data that could be associated with an increased risk of invasive growth, although they do not achieve statistical significance in multivariate analysis, like for example colonic chicken skin mucosa, the presence of erythema over the lesion and the convergence of folds to the lesion.

\subsubsection{Delimitation the edges of the lesion}

The most experienced endoscopists suggest that it is essential to resect at least 1-2 $\mathrm{mm}$ of healthy edge to guarantee a complete resection of the lesion and to reduce the risk of 
recurrence. Some studies also suggest that ablation the edges of the lesion with argon plasma would reduce the risk of recurrence. However, other authors state that removing the entire lesion and including a small portion of healthy tissue in the surgical specimen would achieve the same result.

When an en bloc resection is achieved, this is less important. But when there is a large lesion that requires a piecemeal resection, the burn deforms tissue and it is more difficult to demark the lesion. Although the use of chromoendoscopy and NBI may be useful to detect the remaining tissue, it seems more useful to have a correct demarcation of the lesion before to start the resection. On the other hand, performing submucosal injection for lifting the lesion may distort its appearance by making the edges difficult to identify at the time of resection. Therefore, our standard practice is to define the edges of the lesion with a minimum burn that can be easily seen, which can be performed with argon plasma or with the tip of the polypectomy snare (Figure 5). These burns are easily recognizable; marking the entire periphery of the lesion. They are located at 1-2 $\mathrm{mm}$ from the edge of the lesion and they are separated from each other by 5-6 $\mathrm{mm}$. At the end of resection of the lesion, if none of these burns is visible, we can be sure that we have performed a resection as extensive as originally planned.

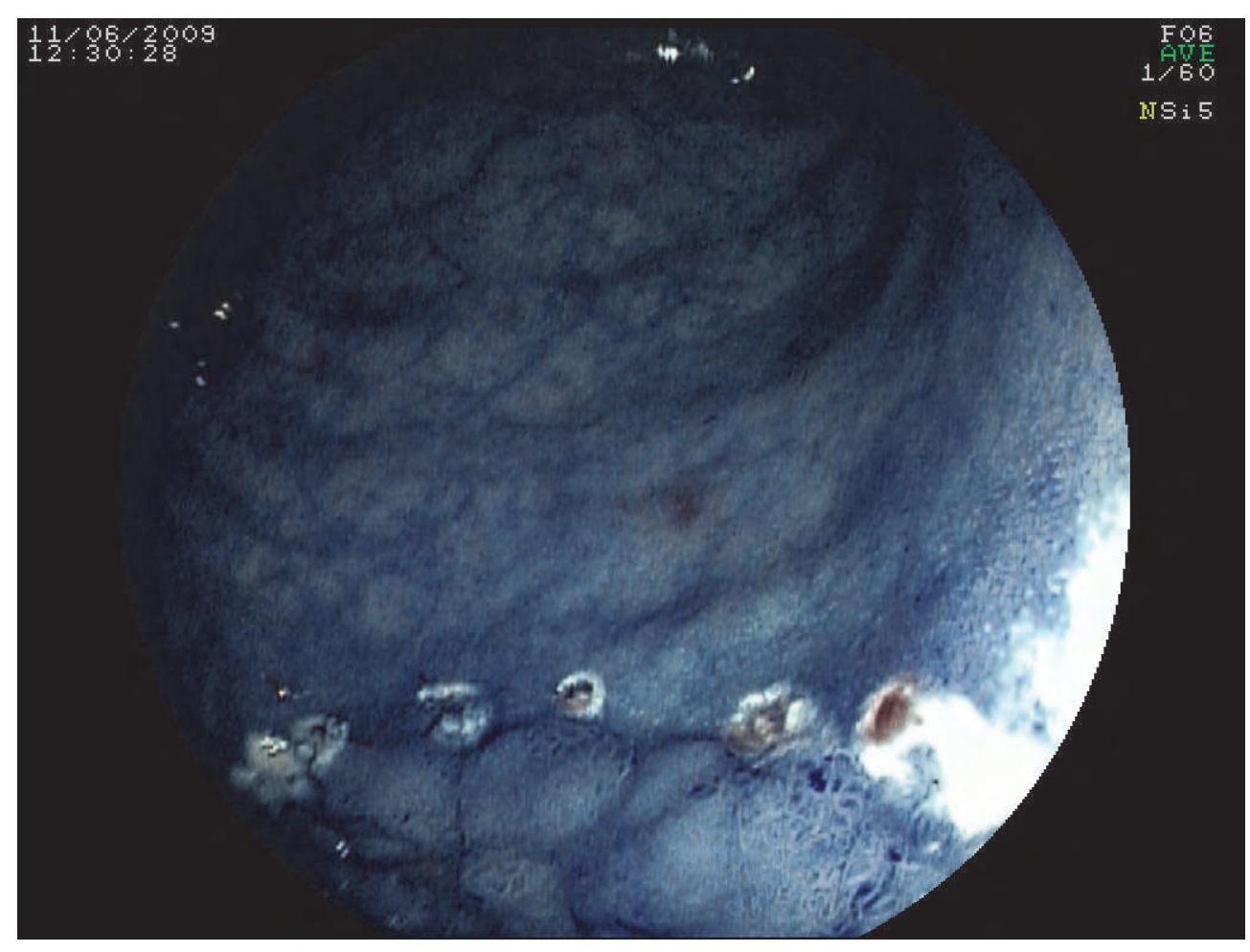

Fig. 5. After clearly limit the edges of the lesion using chromoendoscopy, in this case, computed virtual chromoendoscopy (CVC-FICE). The edges can be marked by burns performed with plasma argon or with snare tip from 3-5 $\mathrm{mm}$ at the edge of the lesion. 
In our center, to correctly identify the edges of the lesion we usually use indigo carmine $0.2 \%$ (Figure 6). In recent years, since we have Computerized Virtual chromoendoscopy (CVC-FICE) (Figure 7) both techniques are used without distinction with identical results.

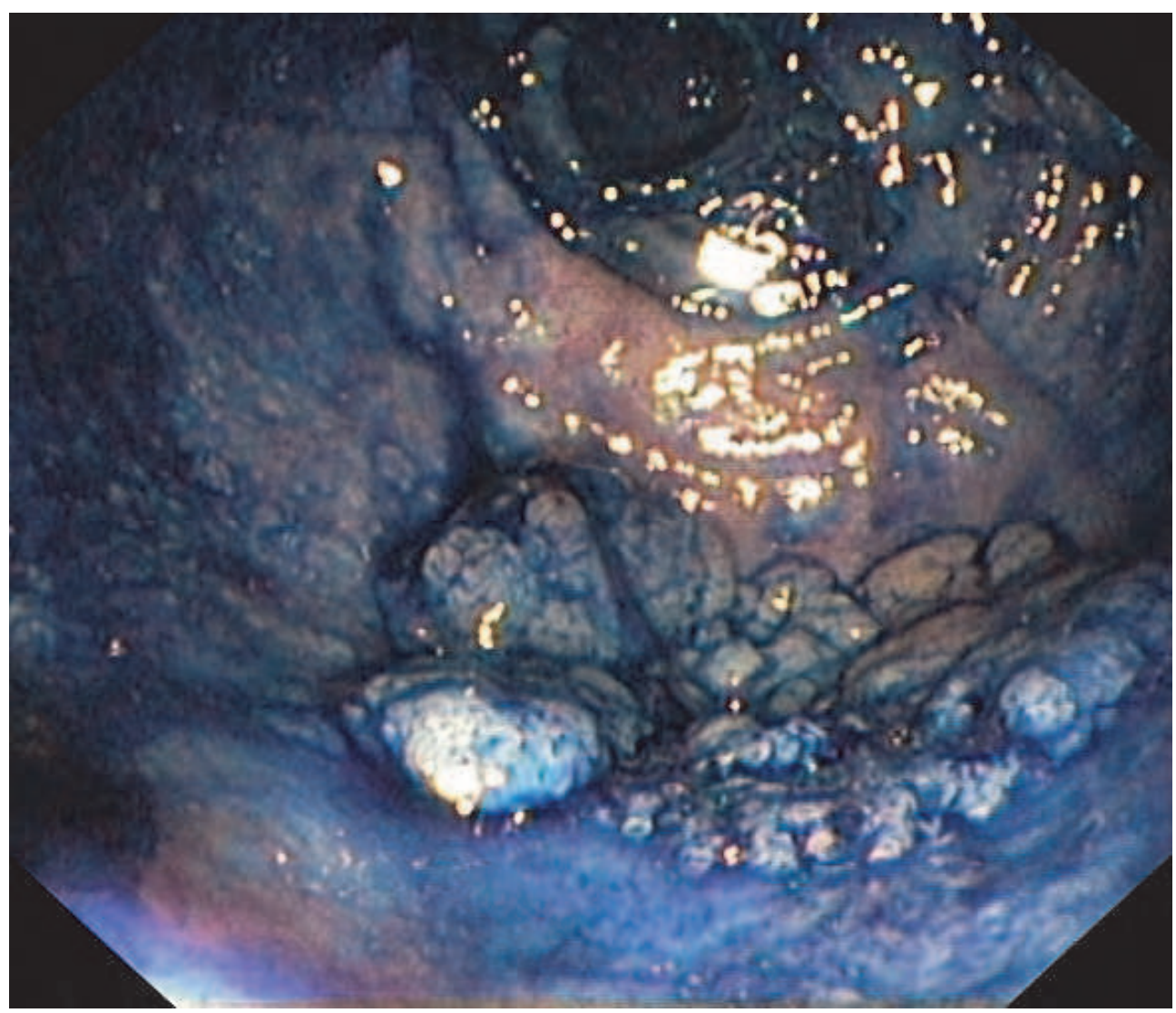

Fig. 6. Flat lesion, stained with $0.2 \%$ indigo carmine, which highlights the surface of the lesion and clearly defines the edges.

\subsubsection{Submucosa injection}

The injection of drugs into the submucosa aims to create a space between the mucosa and submucosa and muscular layer. Thus, resection of sessile or flat lesions is easier, and the risk of complications is reduced, especially perforation. The substance normally used is saline, with or without epinephrine. Since it is an isotonic substance, it spreads quickly, so the lifting disappears, that is why it is important to perform multiple injections, especially in large lesions. We have used other substances, such as glycerol, hyaluronic acid, hydroxypropylmethylcellulose or autologous blood anticoagulated with citrated substances. The lifting they generate is more lasting, allowing a small number of injections. Its major limitation is their high cost so they are no available in most centers. Hypertonic solutions of dextrose $50 \%$ with epinephrine have also been used, with better 
results than normal saline solution, allowing to remove lesions with lower volume of solution injected, fewer number of injections and less time consuming to complete resection. These differences are only significant in case of large lesions. However, it is found a higher frequency of postpolypectomy syndrome that can suggest a possible damage of the colonic wall, probably due to vascular disturbances caused by the hypertonic nature of this solution. It has been recently recommended the use of succilinated gelantine, a very cheap plasma expander, whose only limitation is that it can not be used in patients with hypersensitivity to gelatin. This study asserts that the lift is more lasting and that the number of injections, the total volume injected and the time consumed in the resection are lower. Additionally, the size of the resected fragments is larger and, consequently, lesions of the same size are removed in a smaller number of fragments. Finally, the en bloc resections are almost double that when the lesion is raised just with saline. In our experience, with a saline, methylene blue and epinephrine $1 / 10,000$ mixture most lesions are successfully completely removed. We have recently started to use succilinated gelantine (Gelafundina ${ }^{\circledR}$ ), instead of saline, with good results. Apparently, the lift that it provides is more lasting, requiring less number of injections to complete the resection of the lesions. However, in this moment we do not have enough experience to ensure the superiority of a solution over the classic one.

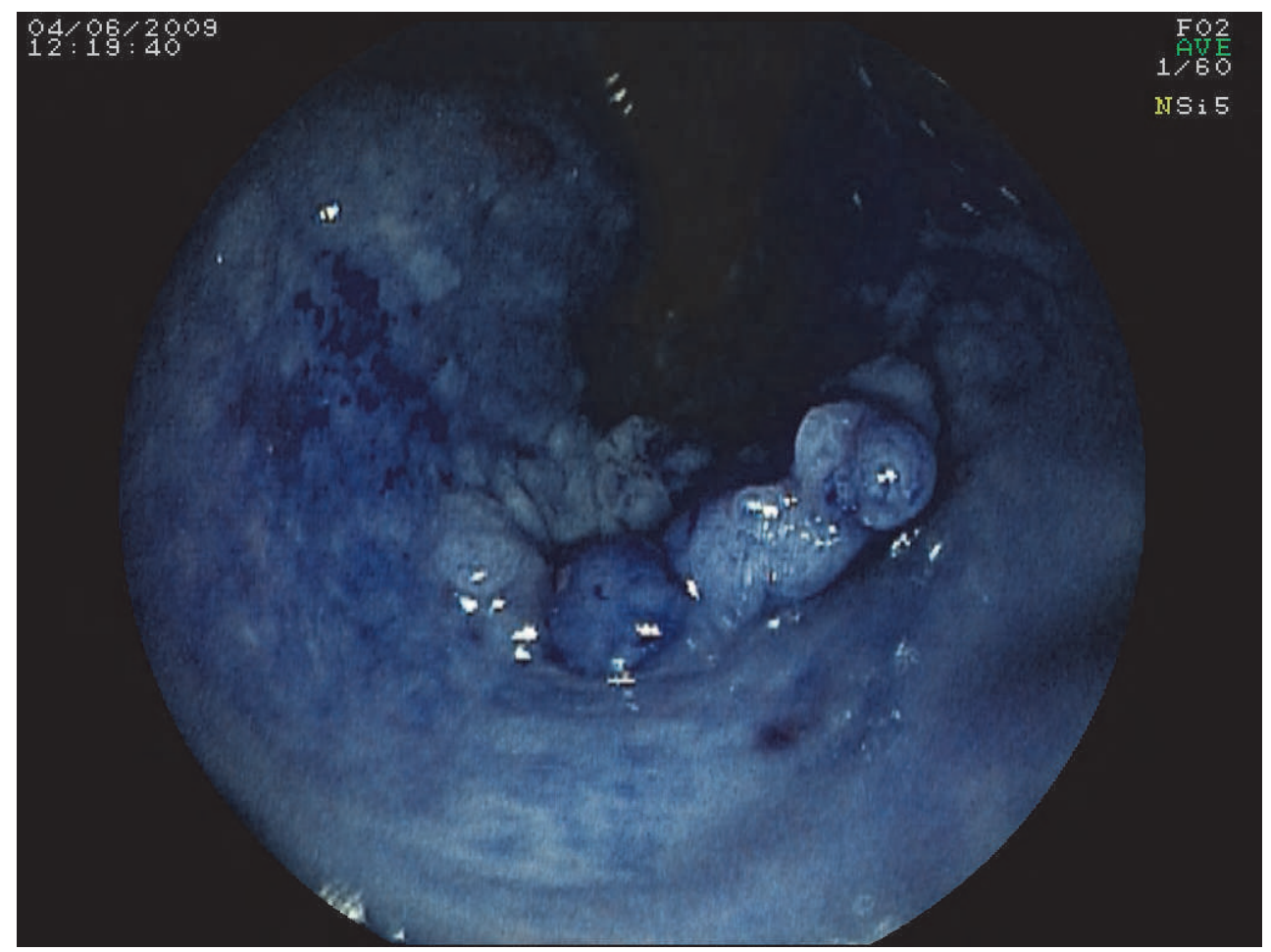

Fig. 7. Computed virtual chromoendoscopy (CVC) is useful for the characterization of colorectal lesions, avoiding the use of coloring agents, catheters and only requiring the use of an endoscope. 
Submucosal injection has to be done very carefully, in small aliquots distributed all over the extent of the lesion to achieve a correct elevation (Figure 8). Especially in lesions located on a fold, it is essential to start lifting the distal part to ensure the lesion gets "closer" to the endoscope, instead of being placed in positions that can difficult the visibility. When a lesion that has not been previously biopsied does not lift ("non lifting sign) it fairly predicts the infiltration of the submucosa by a possible focus of adenocarcinoma (Figure 9). However, biopsies, especially in flat or very small lesions, can cause fibrosis in the submucosa and difficult its correct lift. In the absence of previous biopsies, the "non lifting sign" has a sensitivity of $100 \%$ and a specificity of $99 \%$ in predicting invasive growth. In any case, no elevation of the lesion, just because it is a malignant lesion or because the high risk of perforation, make it reasonable to not perform an endoscopic resection of the lesion.

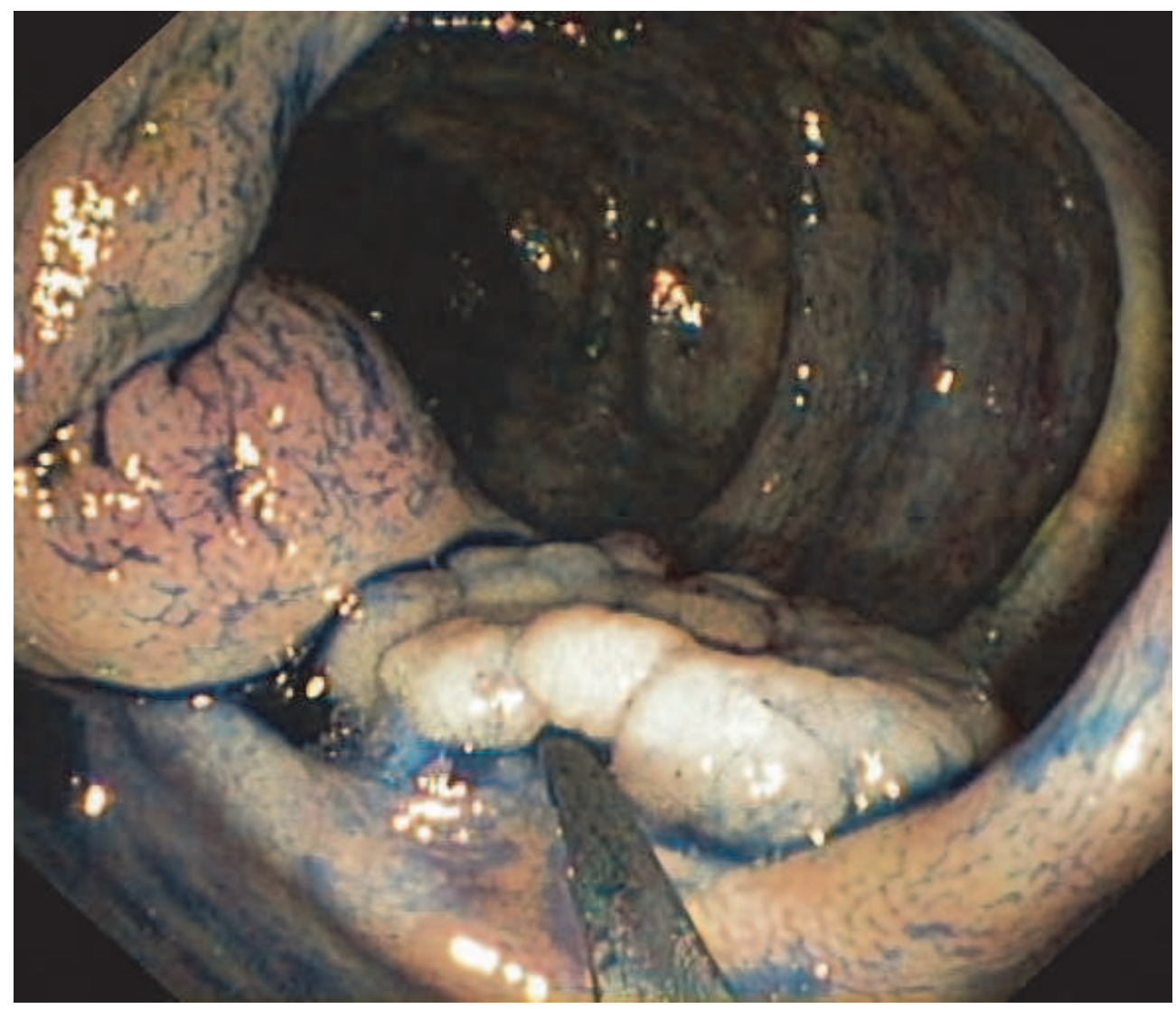

Fig. 8. Submucosal injection, with an adequate lift of the lesion prior to its removal. It is recommended to start injecting at the distal part of the lesion to avoid losing perspective and also to avoid not to see that part of the lesion during the excision. Otherwise, we may be forced to confront the farthest part of it to us in retroversion. 


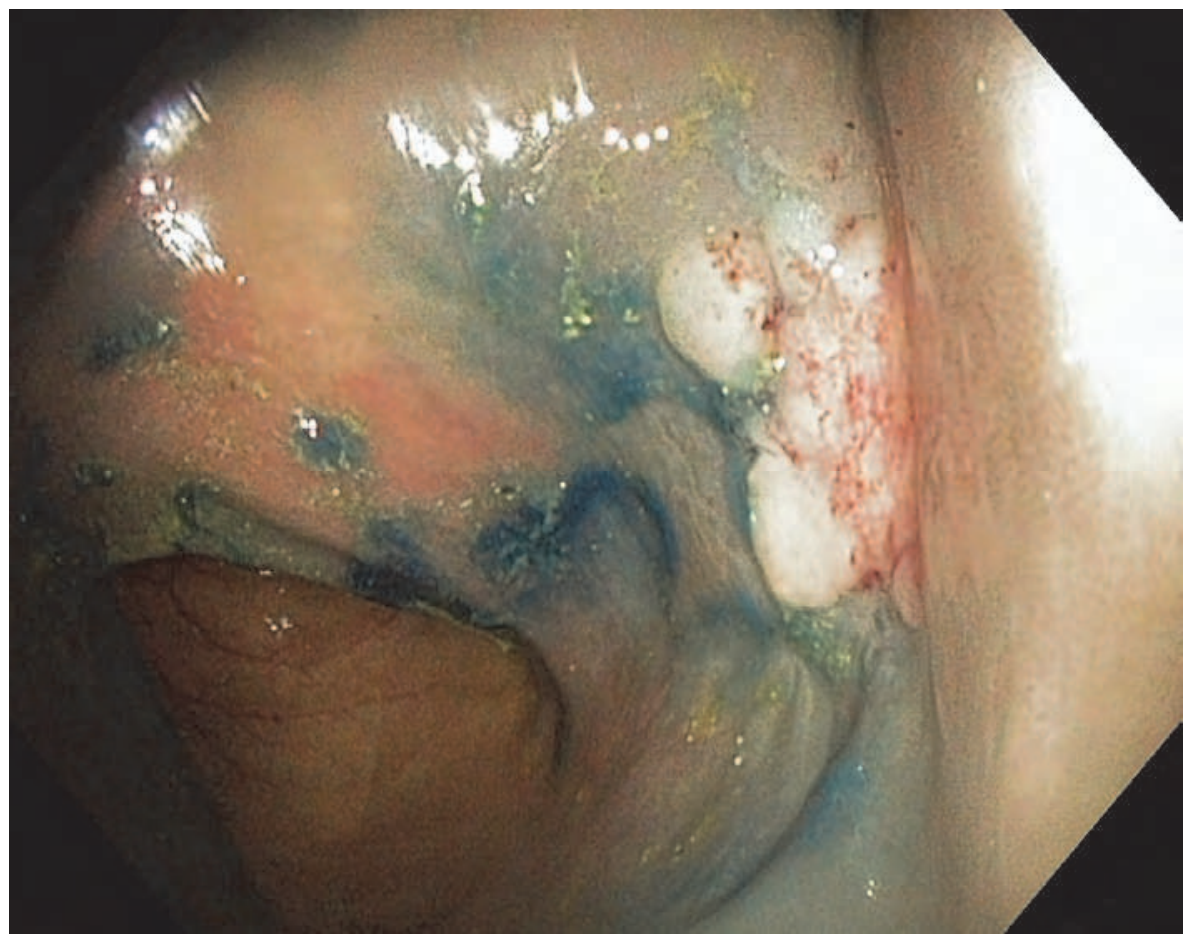

Fig. 9. "Non lifting sign": The lesion is not lifted after submucosal injection. This, if there is no submucosal fibrosis caused by previous attempts of resection, sugests submucosal invasive growth

\subsubsection{Endoscopic mucosal resection technique}

The most commonly technique used is the conventional one consistent on lifting and cutting. After submucosal injection of different substances, the lesion is totally or partly included on a typical polypectomy snare and it is removed in one or more fragments. As we have stated, performing en bloc resection of lesions larger than $2 \mathrm{~cm}$ is neither possible nor desirable, due to the high risk of perforation. If a piecemeal resection is needed, it must be done in the lowest number of fragments as possible in order to restore and "reset" the lesion as close as possible to reality. Thus resection can be achieved for the vast majority of the lesions identified in the colon. Even in a study presented by experienced endoscopists, remarkable results were achieved without making any submucosal injection. It is essential to keep a carefully systematic resection to prevent the persistence of viable tissue areas at the confluence of the different fragments resected. Some authors suggest the possibility of APC ablation of this tissue to prevent recurrence of the lesion, although this practice could increase the possibility of developing postpolypectomy syndrome. Some authors also suggest coagulating the edges of the lesion with APC, thus reducing the risk of recurrence. However, in opinion of leading experts and also ours, this is not necessary if you perform a wide resection including at least 1-2 $\mathrm{mm}$ of healthy mucosa in the resected specimen.

In general, with this simple technique of lifting and cutting, most authors report a technical success rate above $90 \%$. That is, with a simple technique we can achieve resection of most 
lesions. However, some authors have reported small series of cases in which the resection of colorectal lesions is achieved with great success and low risk of complications, using suction and cutting techniques, especially Cap assisted mucusectomy. In our opinion, on a regular basis, it would not be necessary to add complexity to a simple and effective technique in most situations; although this might be a good alternative in case of lesions where the loop can not be placed over them. To facilitate the inclusion of the lesion inside the loop it is often useful to suck as much gas as possible, almost to collapse the lumen. This causes lower intestinal wall tension and this facilitates catching the lesion.

Some authors, in both animal and patients models, have suggested making a circumferential incision at the edges of the lesion before using the snare, allowing it to be snared, thus achieving en bloc resection of large lesions in lesser time. In animal models specimens have been achieved up to $50 \mathrm{~mm}$ in diameter, excised by en bloc resection, without a great risk of perforation. This technique, known as IT-EMR (EMR-incision technique) or as CSI-EMR (circunferential submucosal incision-EMR) could be an alternative to ESD as a tool to achieve en bloc resection of some lesions.

For resection of these lesions we usually use mixed stream, cutting and coagulation, with the Endo-cut system. We use a $120 \mathrm{~W}$ cutting power in rectum and left colon and $80 \mathrm{~W}$ in the right colon, and a coagulation power of $60 \mathrm{~W}$ in both right and left colon. When argon plasma is needed, we use power 100W and a flow of 2 liters / min in rectum and power $60 \mathrm{~W}$ and a flow of 1 to 1.2 liters / min in right colon. Some authors have suggested that the use of pure cutting power causes lesser thermal artifact on the specimen, facilitating the study and a more accurate pathological staging in case of lesions with an infiltrative growth pattern. This would increase the risk of bleeding, which could be controlled using hemoclips as a preventive treatment.

It is essential to complete successful resection of the lesion performing it in a single session. The removal of the lesions, the coagulation of the scar produces fibrosis that would make impossible to lift the lesion in a second time, adding an increasing risk of complications. If in consecutive endoscopic examinations adenomatous tissue is detected, it has to be removed. However, in these cases, the elevation of the lesion with submucosal injection is usually very difficult, and due to the submucosa fibrosis it usually spreads to the lateral areas, lifting more healthy mucosal tissue than residual adenomatous tissue. In one of the largest series published, which belongs to an Australian multicenter group, including 479 patients, it was established an eradication rate of lesions in a single session of $89.2 \%$. Failure factors were involvement of the ileocecal valve by the lesion and the existence of a previous attempt to resection. Submucosal injection is considered an attempted resection, even without resection just because submucosal injection results in submucosal fibrosis. In this case, the probability of failure in the resection is multiplied by 6 . In the same series, it is established as predictors of relapse of the lesion an initial size greater than $40 \mathrm{~mm}$ and the need of using APC to eradicate adenomatous tissue.

\subsubsection{Medium and long term results}

Large lesions $(>20 \mathrm{~mm}$ ) can rarely be en bloc resected, with an estimated recurrence rate of $20 \%(0-52 \%$ in different series published). However, in very large series recurrence rates of lesions have been reported below $10 \%$.

To ensure the success of the procedure, it is essential to perform a systematic resection of the lesions, but also a strict monitoring system of the scars, especially when a piecemeal resection has been carried out. Most authors recommend a first review of the scar 3 months after resection. . In case of detecting residual tumor tissue, resection must be attempted and, 
if it is not possible, we should apply argon plasma. We perform a review and biopsy of the scar at 3 months after initial resection. We perform quarterly reviews until the scar biopsies are negative, making then a review after 6 and 12 months. If after one year of the last examination in which neoplastic tissue was removed there is no evidence of recurrence we consider that we have achieved the cure of the lesion. Some authors believe that a NBI, chromoendoscopy or magnification endoscopy study could be performed to review the scar, so the presence of residual tumor could be ruled out fairly confidently. We combine the study with NBI and biopsy of the scar, especially in large scars where biopsies may not be representative of the entire surface of it. As recently reported normal appearance of the scar and negative biopsies, performed three months after resection, predict with a negative predictive value of $97.4 \%$ freedom from relapse during follow-up. On contrast, it is the size of the lesions $(>4 \mathrm{~cm})$, the most strongly factor associated with a possible recurrence of the lesion. In recent years the term late relapse has been established to define those relapses that can be seen after one or more revisions with negative biopsy scar. In some series, they represent more than half of all recurrences, so patients must be followed for long periods of time. This may be a limiting factor, because if it is impossible to make a correct follow-up this may be the cause of the failure. Therefore, the need for repeating colonoscopy several times is something that should be clearly discussed with the patient before make the decision to choose this form of treatment.

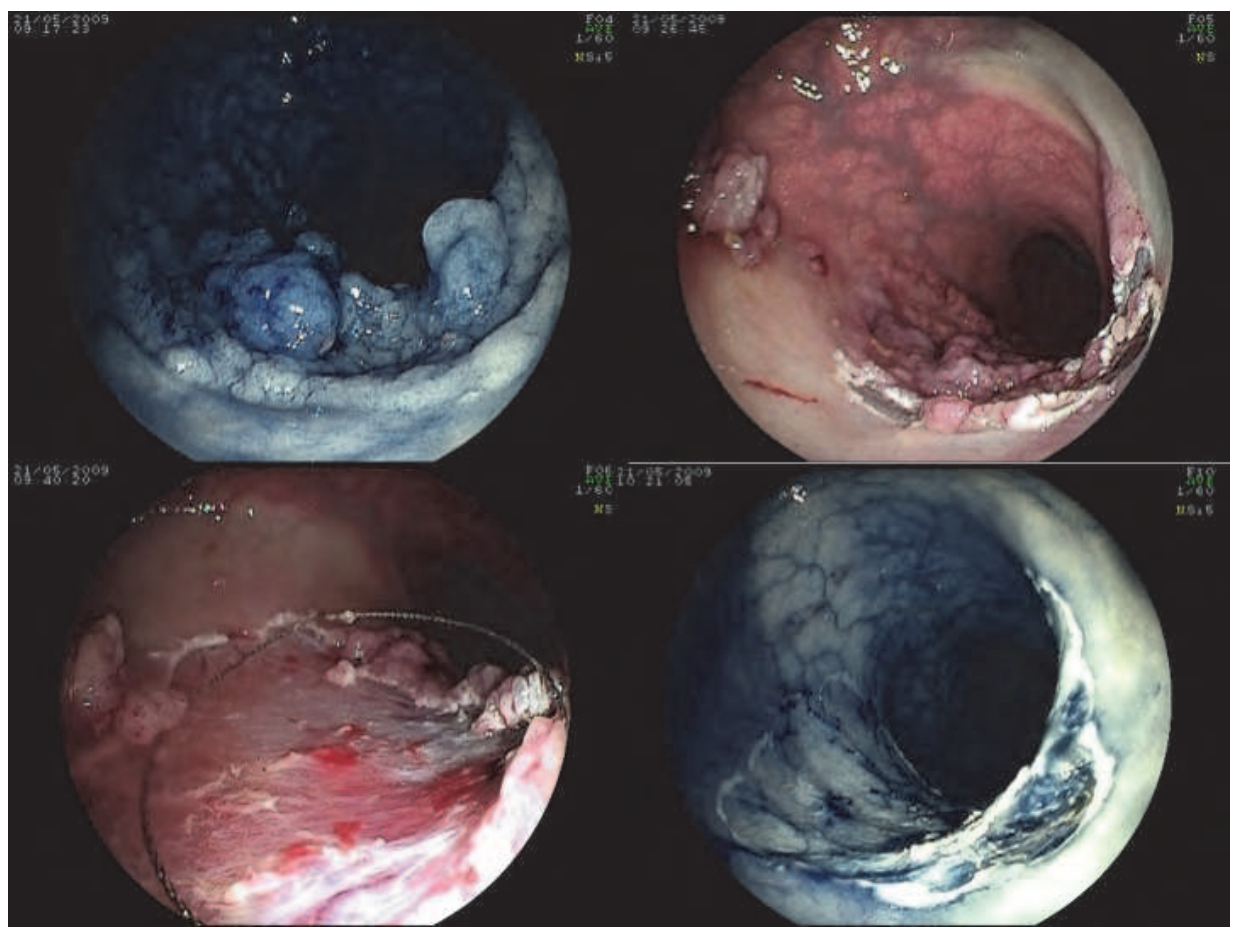

Fig. 10. Piecemeal resection of a large lesion of $6 \mathrm{~cm}$ in diameter, located in the upper rectum. After finishing the resection, CVC is used to check the absence of residual adenomatous tissue in the scar. 
The results of endoscopic resection of large lesions, make that this could be the best therapeutic approach in properly selected patients. The development of laparoscopic techniques for segmental colon resection has questioned the usefulness of such complex endoscopic procedures. There is no doubt that in patients with extremely complex lesions, where a complete resection is not possible, like in case of lesions involving the appendiceal orifice, those affecting two consecutive folds, or those that fail to raise properly, are serious candidates for surgical treatment. However, we must not forget that the surgical resection, even in case of laparoscopic surgery, it is not free of morbidity and mortality, especially in patients with high surgical risk. Secondly, cost of laparoscopic surgery and hospitalization are significantly higher than the cost of endoscopic procedures, even if it is necessary to perform several colonoscopies during follow-up period. Obviously, if endoscopic treatment represents a serious alternative to surgery, it must be safe and effective, so it is necessary to concentrate the most complex cases in expert hands. This is especially interesting in rectum, where the risks of endoscopic resection are lower and potential consequences of surgery are greater. Thus, an Australian group was able to avoid surgery in $90 \%$ of patients that were referred to them from centers where endoscopists had refused endoscopic resection, and they achieved a saving of $\$ 7,000$ per patient that could be endoscopically managed.

\subsubsection{Complications}

The most common complications of endoscopic resection of colorectal lesions, regardless complications due to sedation when performing endoscopy, are bleeding and perforation of the intestinal wall. Bleeding is the most common complication. It is estimated that it occurs in 1 of every 100 procedures. Proper lift of the lesion allows us to avoid the section of the thick vessels of the submucosa, achieving prevention of bleeding. In case that these vessels get exposed or are partially damaged by resection, its coagulation can be performed with thermal methods (coagulation forceps, APC, heat probe) or mechanical ligation can also been performed with hemoclips; although some authors doubt about the profitability of performing prophylactic coagulation of these vessels. What is important, surely, is to make the cut with sufficient calm to properly coagulate the vessels of submucosa. If bleeding occurs, immediate treatment must be done with the same methods described to prevent this complication. Once bleeding is controlled, resection can be continued. We must avoid, obviously, the contact of the polypectomy snare with metal clips, as they can transmit power through the colonic wall and perforate it in the area where they have been inserted. In most cases bleeding is controlled immediately, having no impact on patient. However, bleeding is not always immediate as it can be deferred (between the 24 hours after the procedure, even up to 14-21 days later). In this case, patient usually presents anemia, so admission and blood transfusion can be required.

Perforation is less frequent, but a much more serious complication. It happens when in a fragment of the lesion that has to be resected it is included the muscular layer or the whole wall, damaging it. It can produce a frank perforation or simply the interruption of the muscular layer. To prevent this complication is essential to make a correct lift of the lesion, allowing us to obtain a cutting plane large enough to avoid the muscular layer. It is also essential to recognize the "non lifting sign" because if the lesion is not correctly raised we should not proceed to resection. Once the lesion is inside the loop it can be useful to make traction of it towards the intestinal lumen, achieving to separate the cutting plane from the muscular layer. We can also liberate the muscular layer, if it has been trapped, making soft forward and backward movements. It is also recommended to to suck as much gas as 
possible because this causes lower intestinal wall tension. Most authors also recommend, as a preventive measure, to avoid performing multiple resections of large lesions located on the same place. Finally, it is essential to be extremely careful when removing lesions located on a fold, because its section results in transmural resection. Sometimes, it is difficult to fully recognize the situation of the fold as the anatomy of the colon can be altered by the lesion and submucosal injection.

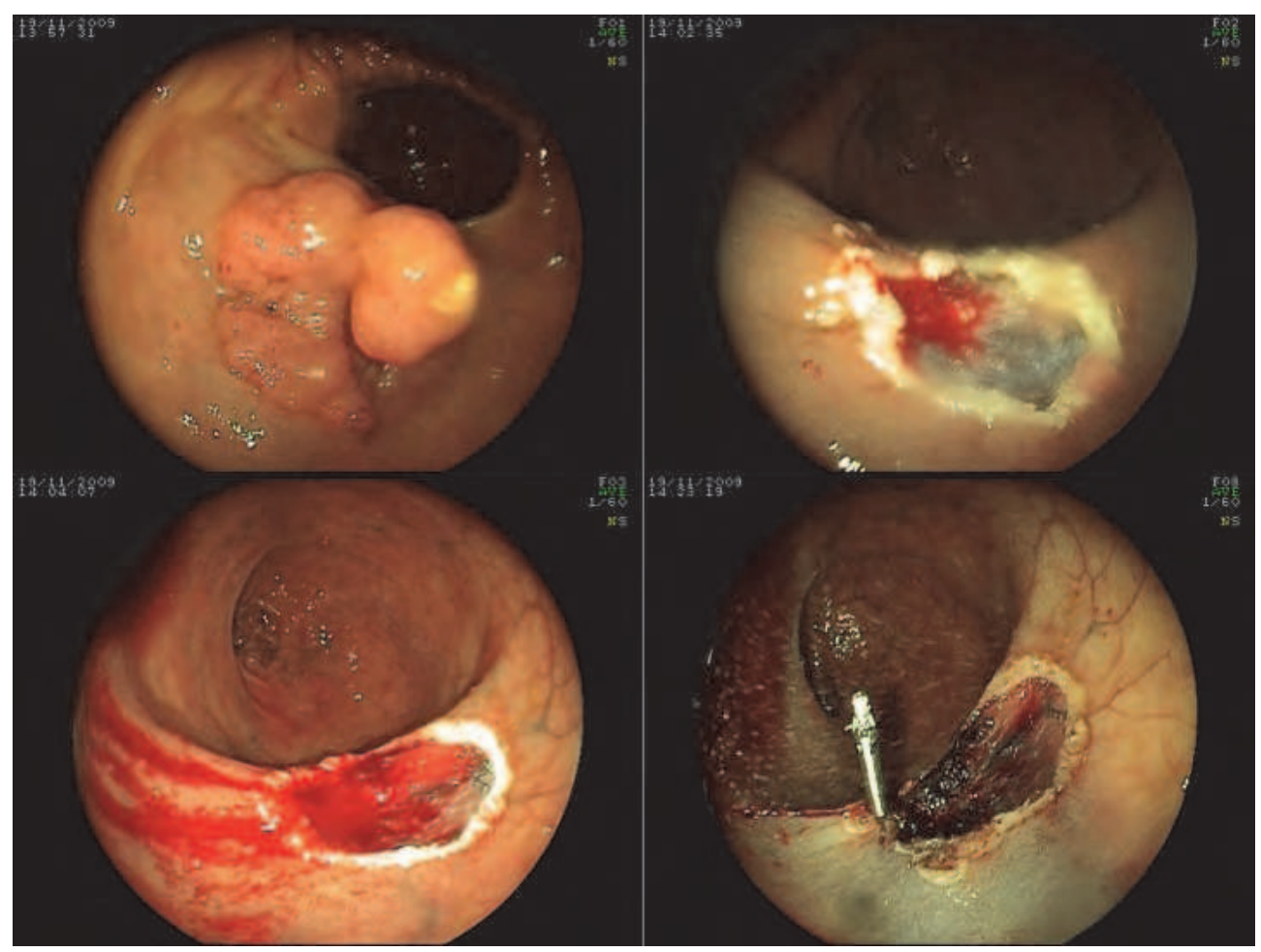

Fig. 11. Resection of a large lesion in the sigmoid colon with hemorrhage during the procedure, controlled by the placement of several hemoclips.

Perforation can occur immediately, being recognized during the procedure so it can be directly solved, not having serious consequences for the patient. On other occasions, perforation can be deferred or delayed, produced by a spontaneous "rupture" of the wall under a large scar. In these cases the colon is not clean, so the patient would present symptoms. It usually requires systematically surgical repair, and it is usually needed to perform a temporal colostomy. In case that perforation is detected during resection of the lesion it can be endoscopically managed, covering the defect by suturing using hemoclips. Modern Ovesco devices ("over the scope hemoclips ") or different tools to perform endoscopic suturing could allow closing large defects. Indeed, the possibility of transmural sutures would allow closing large defects, providing a security level that would allow endoscopists face larger or more complex lesions. If there has been a transection of the muscular layer without frank perforation, this can be sufficient, but if there is a complete 
perforation of the wall, it is usually necessary to restrict oral intake, to establish broadspectrum antibiotics and, depending on the magnitude of the pneumoperitoneum, to drain it. In most cases, we can solve problem and so avoid surgery. Consequently, it is essential to immediately detect the complication. Some authors define "target sign" as a whitish lesion on a muscular layer stained in blue (methylene blue) and "mirror target sign" on the surgical specimen that includes a section of the muscular layer. If this sign is observed, we should act accordingly.

Sometimes there is a transmural burn in the colonic wall, without frank perforation. The patient can present abdominal pain and signs of peritoneal irritation, but in the absence of pneumoperitoneum. This is called postpolypectomy syndrome. Conservative management, based on analgesics, broad spectrum antibiotics and fluid therapy is usually enough.

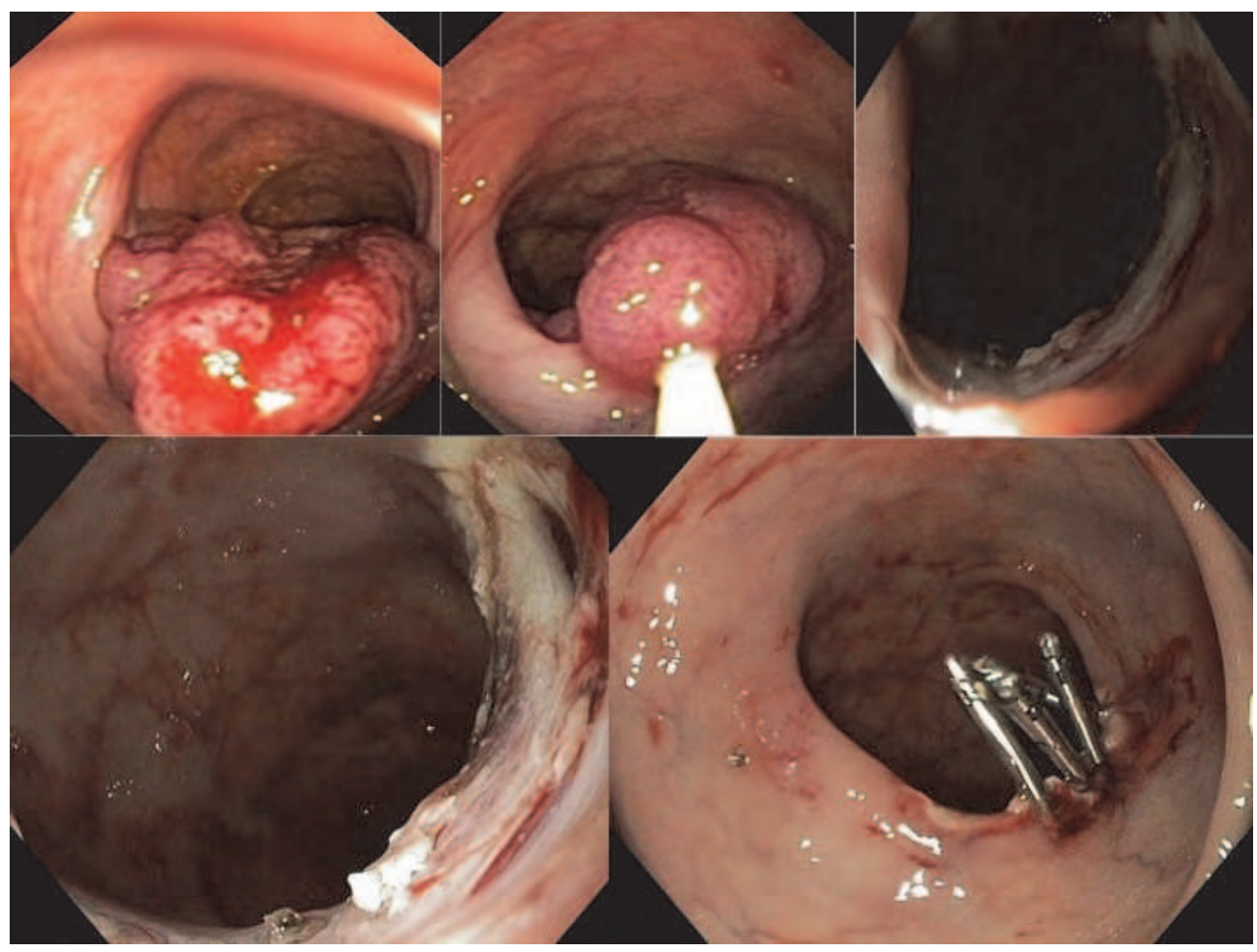

Fig. 12. Resection of large sessile lesion in sigmoid colon. After completing the resection it is clearly seen a lesion of the muscular layer, approximately $10 \mathrm{~mm}$ in length, which is completely closed by applying hemoclips. The patient remained in hospital during 48 hours and after restarting oral intake he was discharged without complications.

Resection of large colorectal lesions involves performing submucosal injection through a non-sterile system. In addition, a huge defect in the mucosa will persist over a long time. Both situations could represent the entry point for infections. However, it has not been observed a significant incidence of bacteremia after these procedures, so it is not justified a systematic antibiotic prophylaxis. 
In conclusion, complications are rare on endoscopic resection of large colorectal lesions and, in most cases; these can be solved in a conservative way, endoscopically.

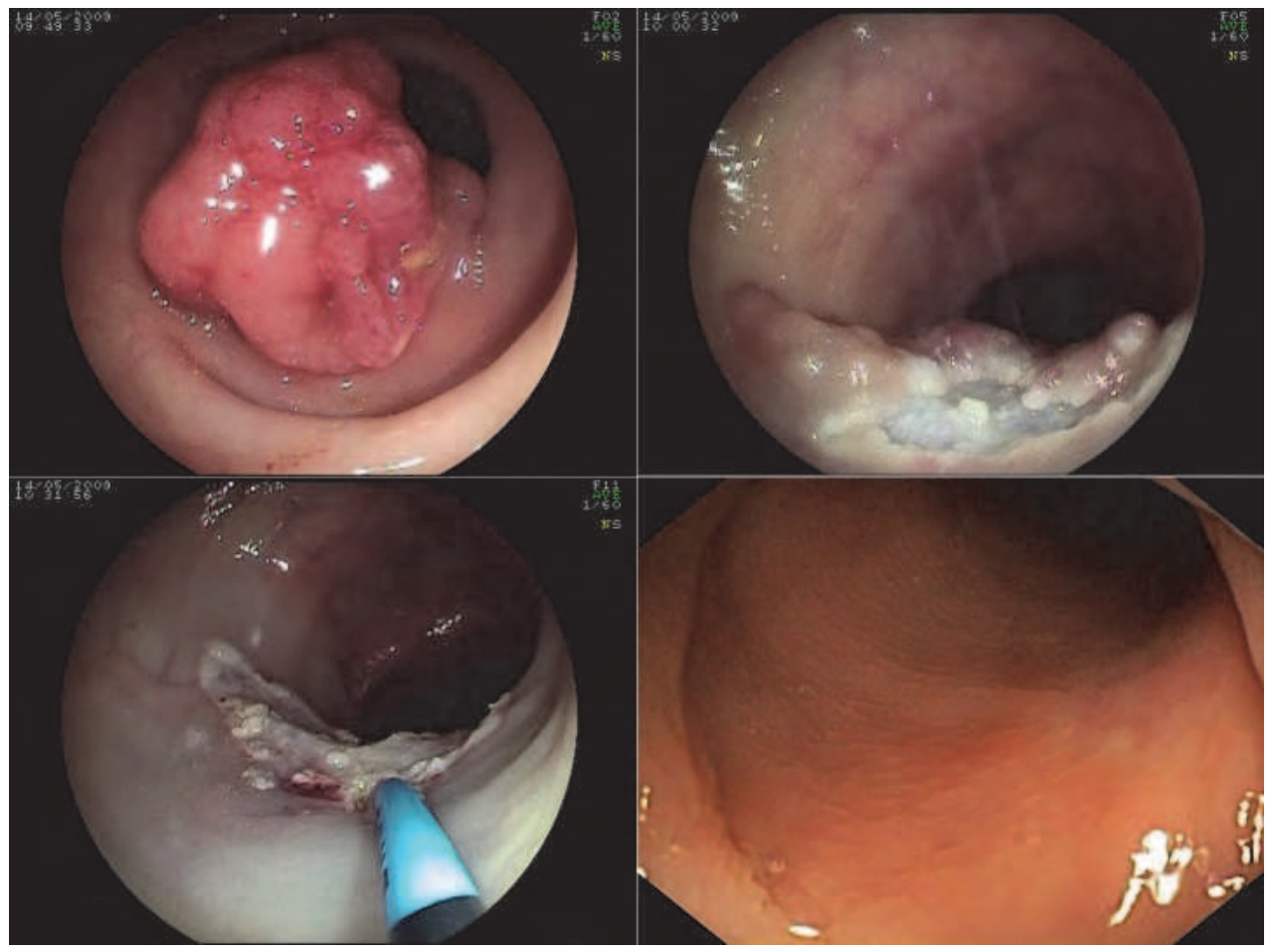

Fig. 13. In the picture we can visualize the piecemeal resection of a large sesile lesion, in the distal sigmoid colon. In this particular case we completed the resection with the coagulation of the lesion edges with argon plasma. In the last image, we can see the scar of the lesion, without recurrence.

\subsection{Endoscopic Submucosal Dissection}

Resection of large lesions generates uncertainty in the physician and patient, especially in those that are more likely to have invasive growth. En block resection of the lesions results in more certain of achieving a complete resection, a lower recurrence rate, and facilitates a better interpretation of the spcimen by the pathologist, achieving a correct classification of lesions that present an infiltrative growth.

The Endoscopic Submucosal Dissection (ESD) is a technique originally designed for the resection of gastric lesions, but later it was adapted to esophageal lesions and ultimately it was used for en bloc removal of large colorectal lesions. The great attraction of this technique is that, in fact, it allows the resection of large lesions in a single piece. The great inconvenient is its technical complexity, with an increased risk of complications, so its use in the Western countries is quite restricted.

As it has been already explained, it is in lesions that are more likely to have an invasive growth where it is more important to perform an en bloc resection. Then, the pathologist 
would be able to study the whole specimen, to perform accurate staging and, consequently, to establish the cure of the lesion or the need for surgery. It is considered that endoscopic removal of a lesion presenting adenocarcinoma with infiltration of submucosa can be curative if the following conditions are present:

- Well or moderately differentiated adenocarcinoma

- Absence of lymphatic and vascular permeation

- Presence of intramucosal adenocarcinoma

- Adenocarcinoma that infiltrates submucosa, but only in the most superficial portion (invasive growth of no more than 1000 microns from the muscularis mucosae)

- $\quad$ Edges of resection free of lesion

Endoscopic resection can be considered curative if lesions fulfil these conditions because the risk of lymphatic metastasis is minimal.

Given this, it could be said that indications of ESD in colon could be the following:

- Colonic polyps or lesions presenting intramucosal adenocarcinoma

- $\quad$ Early Neoplasms IIa (Paris classification) with a diameter less than $2 \mathrm{cms}$

- $\quad$ Neoplasms IIb IIc (Paris classification) with a diameter less than $10 \mathrm{mms}$

- $\quad$ Laterally spreading tumors

\subsubsection{Endoscopic Submucosal Dissection technique}

The procedure is very similar, in the majority of the phases, to endoscopic mucosa resection. It begins, as it is logical, with a correct identification, visualization, demarcation and characterization of lesions. Everything explained in EMR is valid now. Using chromoendoscopy, NBI and all the available technology are helpful. The characterization of the lesion according to morphology, Paris classification, and crypts pattern should lead us to the final decision to proceed or not to perform endoscopic resection and also to choose the most appropriate technique. After considering endoscopic treatment, firstly we have to demark the edges of the lesion in the same way as for EMR. In this case, submucosal injection is more important than in EMR, since it is necessary to achieve a good level of separation of the outer layers of the wall respecting to the muscular layer to avoid injury. For this reason, glycerol or hyaluronic acid are preferable because they remain longer in the submucosa. Then, a circumferential incision is performed, which isolates the lesion from the rest of the surrounding mucosa and it allows to open a space to introduce the endoscope and finally to start the dissection. This incision can be made using a conventional needle knife or material especially designed for that purpose. The placement of a transparent cap on the tip of the endoscope is essential to facilitate the visibility at the submucosa level and to permit the management of the material used for dissection. Finally, the lesion is dissected until complete the resection of the lesion in only one fragment.

Various instruments have been designed for the dissection of the submucosa, all of them with common and differentiating elements. They all have advocates and detractors, and each expert recommends the use of the one that best suits to the way of working of the endoscopist. However, experts also recommend mastering more than one of them, because in some lesions an instrument can be adapted better than another. Today there is a wide variety of instruments available: the IT knife (isolated tipped knife), the flex knife, the hook knife (needle point hook-shaped) and the triangle tip knife (triangular-tipped). In addition, recently new instruments have been designed such as the flush knife, which allows submucosal injection and cutting with a single device, allowing saving time by not having to exchange instruments. 
In addition, there are specially designed coagulation forceps to coagulate vessels in the submucosa, either as bleeding prevention or as treatment. However, coagulation of vessels can be performed with the same cutting instrument, as long as there is a good domain of the technique.

\subsubsection{Medium and long term results}

In Japan there are already quite large series of colorectal neoplasms removed by ESD. In all cases, they are great experts in the technique, with many hundreds of procedures performed in the upper gastrointestinal tract. In all cases the results are quite similar. The en bloc resection rate is $85-94 \%$, with a cure rate of $80-90 \%$. As expected the en bloc resection rate is clearly superior to the one achieved with the conventional mucusectomy, and the cure rate is also slightly higher, not being necessary in this case to perform several sessions to complete the treatment of the lesion. In contrast, as expected in a more technically demanding and complex procedure from a technical standpoint, the time spent on each patient is significantly higher than used in EMR.

\subsubsection{Complications}

Complications, both in the case of perforation or bleeding, are considerably higher in the ESD than in EMR. This fact, together with the time consumed by each procedure, makes that the technique has not spread too much in Europe. If in upper gastrointestinal tract the number of procedures performed in Europe is reduced to a few hundreds of patients, in the colorectal area, theoretically more complex due to the reduced thickness of the wall and with an increased risk of complications, the number is even lower.

Complications related to ESD are the same as for EMR, but considerably more frequent, even in the case of Japanese experts. After reviewing the different series there is a perforation rate of around $5 \%$ and bleeding occurs with a frequency of 2-3\%. In most cases, as in EMR, these complications can be resolved by endoscopic treatment, being exceptional the need to refer the patient for surgery to solve the complication.

\section{Conclusions}

The vast majority of benign lesions of the colon and lesions that present malignant degeneration, but without a massive infiltration of the submucosa by cancer, can be endoscopically managed and endoscopic resection may be considered curative. However, endoscopic resection of these lesions is not always simple and sometimes it requires great technical skill and perfect mastery of technique and material used. In addition, it is essential to properly master the different hemostatic techniques as well as the available devices to close perforations in order to be able to solve complications that could happen during the procedure. The security and confidence in resolving complications, makes these less feared, so indications for endoscopic procedures would be extended.

ESD and EMR are complementary techniques in colorectal disease. ESD is much more complex and it is associated with a greater number of complications and requires considerably more time consuming. EMR is easier, and involves the use of material with which the endoscopists are usually familiar; it presents a lower risk of complications but in contrast, it presents a major limitation in en bloc resection of large lesions. However, EMR can keep its place: we believe that it is the ideal technique for resection of all those lesions that can be en bloc resected using this technique and it may not be unreasonable to perform 
a piecemeal resection of those lesions with very low risk of developing invasive growth (laterally spreading tumors with granular surface). By contrast, those lesions more likely to present invasive component always without breaking the clearly established indications for endoscopic resection and fulfilling the curative criteria of lesions, should be resected using the ESD.

Finally, as we have seen, the adecuate selection of patients for the endoscopic procedure and its results, require a great domain of it. This is only possible if the management of these patients is carried out in the context of specialized units, by expert endoscopists and with special dedication to this type of pathologies.

\section{Acknowledgements}

We especially thank the whole team of the Endoscopy Unit of the Complejo Hospital Universitario de Vigo.

\section{References}

Khashab M, Eid E, Rusche M, Rex DK. Incidence and predictors of "late"recurrences after endoscopic piecemeal resection of large sessile adenomas. Gastrointest Endosc. 2009; 70:344-9.

Conio M, Ponchon T, Blanchi S, Filiberti R. Endoscopic mucosal resection. Am J Gastroenterol. 2006; 101: 653-63.

Kaltenbach T, Friedland S, Maheshwari A, Ouyang D, Rouse RV, Wren S, Soetikno R. Shortand long-term outcomes of standardized EMR of nonpolypoid (flat anddepressed) colorectal lesions $>$ or $=1 \mathrm{~cm}$ (with video). Gastrointest Endosc. 2007;65:857-65.

Swan MP, Bourke MJ, Moss A, Williams SJ, Hopper A, Metz A. The target sign: an endoscopic marker for the resection of the muscularis propria and potential perforation during colonic endoscopic mucosal resection. Gastrointest Endosc. 2011; 73:79-85.

Niimi K, Fujishiro M, Kodashima S, Goto O, Ono S, Hirano K, Minatsuki C, Yamamichi N, Koike K. Long-term outcomes of endoscopic submucosal dissection for colorectal epithelial neoplasms. Endoscopy. 2010; 42: 723-9. 


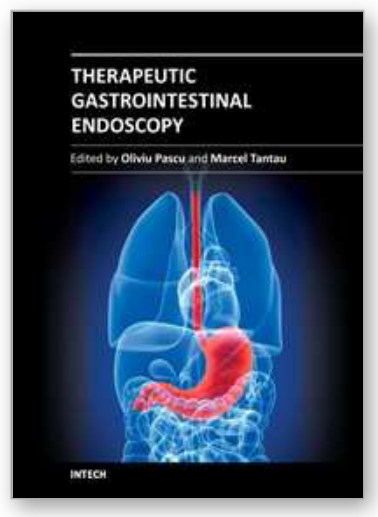

\author{
Therapeutic Gastrointestinal Endoscopy \\ Edited by Prof. Oliviu Pascu
}

ISBN 978-953-307-743-7

Hard cover, 216 pages

Publisher InTech

Published online 22, September, 2011

Published in print edition September, 2011

Endoscopy has had a big role in the development of modern gastroenterology. Modern endoscopy will certainly be more therapeutic. It started with endoscopic hemostasis and polipectomy, than beginning of the 1970 's with the advent of endoscopic sphincterotomy extended to biliopancreatic pathology and has a huge impact in this difficult pathology. Plastic stents made the first steps in endoscopic palliation of neoplastic jaundice, metallic stents, covered or uncovered are better for biliary palliation and can be used also for palliation neoplastic obstruction at different levels of the digestive tube. Resection of digestive tumors has evolved now to sub-mucosal resection, looking to have one-piece complete resection. Interventional endoscopy is now very complex and takes a lot of time for endoscopists to learn properly these techniques. This book is a very good up-to-date overview of new techniques of interventional endoscopy for those who want to learn or develop their knowledges in this field.

\title{
How to reference
}

In order to correctly reference this scholarly work, feel free to copy and paste the following:

David Martinez Ares, Pamela Estévez Boullosa and José Ignacio Rodríguez Prada (2011). Endoscopic Resection of Large Colorectal Lesions, Therapeutic Gastrointestinal Endoscopy, Prof. Oliviu Pascu (Ed.), ISBN: 978-953-307-743-7, InTech, Available from: http://www.intechopen.com/books/therapeuticgastrointestinal-endoscopy/endoscopic-resection-of-large-colorectal-lesions

\section{INTECH}

open science | open minds

\section{InTech Europe}

University Campus STeP Ri

Slavka Krautzeka 83/A

51000 Rijeka, Croatia

Phone: +385 (51) 770447

Fax: +385 (51) 686166

www.intechopen.com

\section{InTech China}

Unit 405, Office Block, Hotel Equatorial Shanghai

No.65, Yan An Road (West), Shanghai, 200040, China

中国上海市延安西路65号上海国际贵都大饭店办公楼 405 单元

Phone: +86-21-62489820

Fax: $+86-21-62489821$ 
(C) 2011 The Author(s). Licensee IntechOpen. This chapter is distributed under the terms of the Creative Commons Attribution-NonCommercialShareAlike-3.0 License, which permits use, distribution and reproduction for non-commercial purposes, provided the original is properly cited and derivative works building on this content are distributed under the same license. 